\title{
Author Correction: Adrenergic nerve degeneration in bone marrow drives aging of the hematopoietic stem cell niche
}

Maria Maryanovich (D), Ali H. Zahalka (D), Halley Pierce, Sandra Pinho (iD, Fumio Nakahara iD, Noboru Asada, Qiaozhi Wei, Xizhe Wang, Paul Ciero, Jianing Xu, Avigdor Leftin and Paul S. Frenette $\mathbb{D}$

Correction to: Nature Medicine https://doi.org/10.1038/s41591-018-0030-x, published online 07 May 2018.

In the version of this article originally published, the key for Fig. $4 \mathrm{c}$ was incorrect. The symbols for 'Sham' and 'Den' were reversed. The error has been corrected in the PDF and HTML versions of the manuscript.

\section{Publisher Correction: Microbial network disturbances in relapsing refractory Crohn's disease}

Bahtiyar Yilmaz DD, Pascal Juillerat (D), Ove Øyås, Charlotte Ramon, Francisco Damian Bravo, Yannick Franc, Nicolas Fournier, Pierre Michetti (D), Christoph Mueller (D), Markus Geuking (D), Valerie E. H. Pittet, Michel H. Maillard, Gerhard Rogler, Swiss IBD Cohort Investigators, Reiner Wiest, Jörg Stelling and Andrew J. Macpherson (D)

Correction to: Nature Medicine https://doi.org/10.1038/s41591-018-0308-z, published online 21 January 2019.

Owing to an error during typesetting, a number of references were deleted from the Methods reference list. This altered all of the references in the Methods section and some of the references in Extended Data Fig. 5, making them inaccurate. References 121-134 were added back into the Methods reference list, and the references in the Methods section and in Extended Data Fig. 5 were renumbered accordingly. The error has been corrected in the PDF and HTML versions of this article.

\section{Publisher Correction: Non-invasive prenatal sequencing for multiple Mendelian monogenic disorders using circulating cell-free fetal DNA}

Jinglan Zhang $\mathbb{D}^{\mathrm{D}}$, Jianli Li, Jennifer B. Saucier, Yanming Feng, Yanjun Jiang, Jefferson Sinson, Anne K. McCombs, Eric S. Schmitt (D), Sandra Peacock, Stella Chen, Hongzheng Dai, Xiaoyan Ge, Guoli Wang, Chad A. Shaw, Hui Mei, Amy Breman D, Fan Xia, Yaping Yang, Anne Purgason, Alan Pourpak, Zhao Chen, Xia Wang, Yue Wang, Shashikant Kulkarni, Kwong Wai Choy, Ronald J. Wapner, Ignatia B. Van den Veyver, Arthur Beaudet, Sheetal Parmar, Lee-Jun Wong and Christine M. Eng

Correction to: Nature Medicine https://doi.org/10.1038/s41591-018-0334-x, published online 28 January 2019.

In the version of this article originally published, some cases that were presented in Fig. 3 should have been underlined but were not. The appropriate cases have now been underlined. The error has been corrected in the print, PDF and HTML versions of the article. 


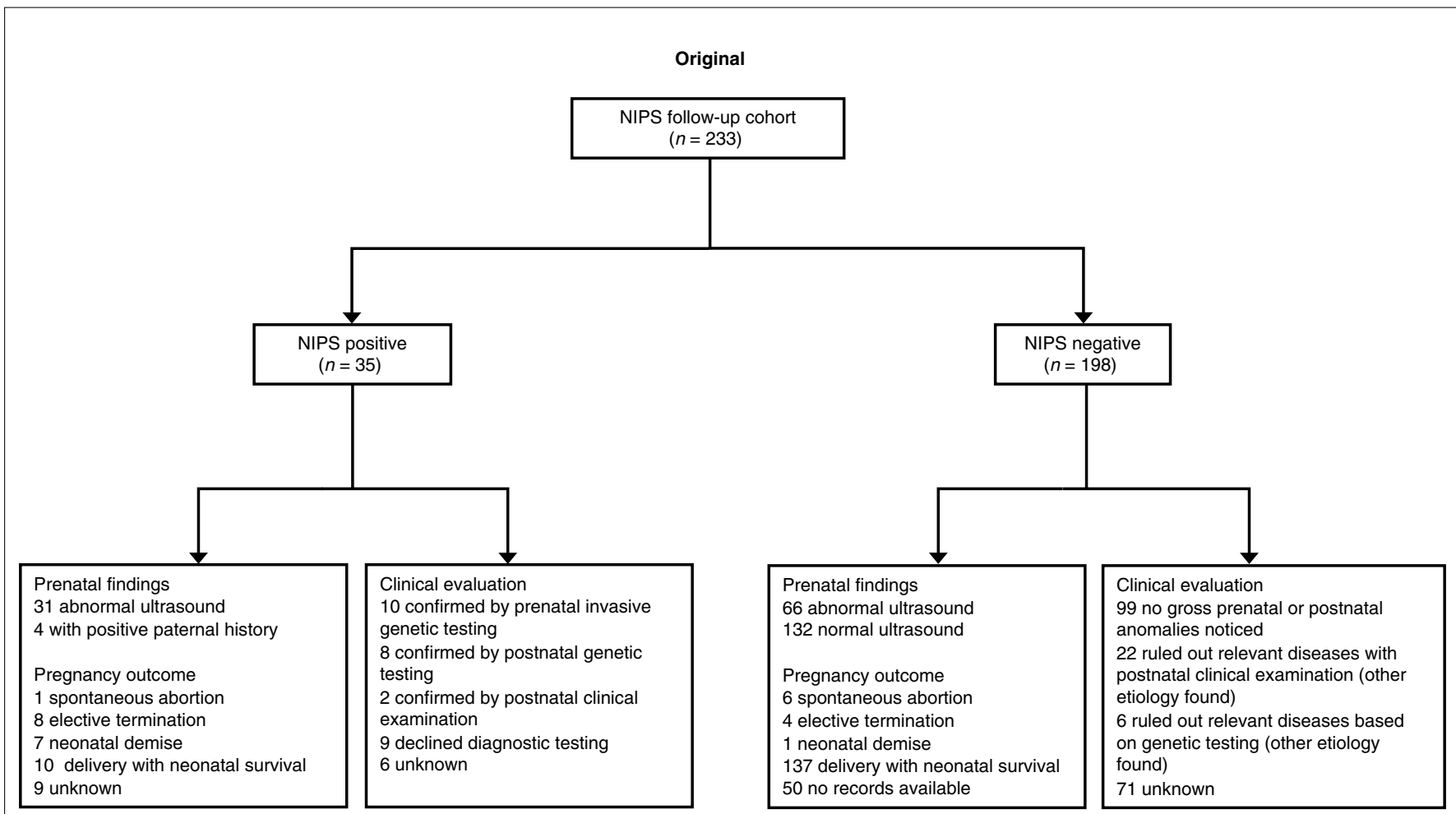

\section{Corrected}

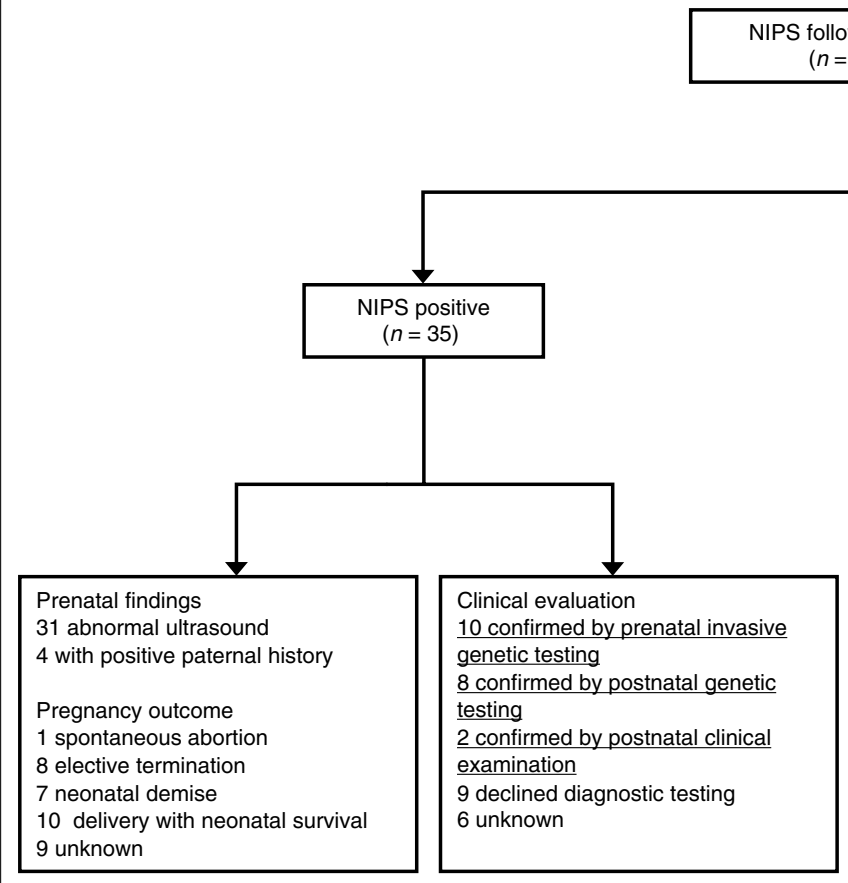

follow-up cohort

$(n=233)$

Fig. 3 | Original and Corrected.

Published online: 20 February 2019

https://doi.org/10.1038/s41591-019-0391-9 